\title{
La siembra de tuna (Opuntia ficus-indica (L.) Miller) en un desierto nor costeño, caso CIPTT - UAP, Mucupe, Chiclayo
}

\author{
Planting of the pear (Opuntia ficus-indica (L.) Miller) in a coastal desert north, if CIPTT - \\ UAP, Mucupe, Chiclayo \\ Julio Bazán ${ }^{1}$, Carlos Urbina ${ }^{2}$
}

http://dx.doi.org/10.21503/CienciayDesarrollo.2014.v17i2.03

\section{RESUMEN}

La siembra experimental de tuna con riego por goteo, se ha desarrollado en el Centro de Investigación Producción y Transferencia Tecnológica de la Universidad Alas Peruanas y se demuestra un rendimiento bueno ( 23 TM por cosecha/hectárea) en terrenos eriazos del desierto norcosteño del Perú .

Palabras Clave: Tuna, adaptación, siembra, riego.

\section{ABSTRACT}

The experimental planting of tuna with drip irrigation has been developed at the Research Center Production and Technology Transfer at the University Peruvian Wings and good performance (23 MT per crop / hectare) norcosteño brownfields the desert of Peru is demonstrated.

Keywords: Tuna, adaptation, planting, irrigation.

\section{INTRODUCCIÓN}

Los restos sobre el uso de cactáceas encontrados en el Perú datan de la época del Horizonte Pre-agrícola (10,000años de antigüedad), y se encontraron en los basurales próximos a la cueva de Pachamachay en las cercanías del lago de Junín sobre los $4200 \mathrm{msnm}$. En ese sitio se hallaron semillas de una especie de Opuntia de una antigüedad de más de 11,800 años, la cual podría tratarse de Austrocylindropuntia floccosa, que es la única que crece en tales altitudes y cuyos frutos aún siguen siendo consumidos en las serranías del centro y sur del país, regiones altas caracterizadas por la prodción controlada o silvestre de esta fruta...

La tuna es conocida como Prickly pear, Cactus pear, Cactus fruti en Estados Unidos; Fico d' India (Sicilia), Figo morisca (Cerdeña), Figo

\footnotetext{
1 Ingeniero agrónomo, Director del CIPTT Mocupe, E-mail: jcbazan.1206@hotmail.com

2 Ingeniero agrícola, Gerente Técnico de la Junta de Usuarios de Zaña. E-mail: curbina_1962@hotmail.com
} 
della barbarie en Italia; Higo en España; chumbo en Francia; Tzabar en Israel; Kaktusfeigen en Alemania; Turksupurug en Sudafica, Nopal en México y Tuna en Perú y Latinoamérica.

El primer nombre español es Higo de las Indias, que alude a su origen, las "Nuevas Indias" y de allí su primer nombre científico: Cactus ficus-indica L. El nombre ficus-indica había sido usado en "frases diagnósticas" ya mucho antes de Linneo, para designar varias especies.

\section{Nombre científico: Opuntia ficus-indica (L.) Miller. \\ Nombre común: "Tuna” (Perú, Chile, Argentina, México) \\ Sinónimo: Cactus ficus-indica $\mathrm{L}$. Familia: Cactaceae}

\section{Ubicación}

En el Norte del Perú encontramos grandes desiertos con miles de Hectáreas y con escasa disponibilidad de agua, por lo que la Universidad Alas Peruanas dispuso un trabajo experimental de sembrar Tuna en el centro de Investigación Producción y Transferencia Tecnológica (CIPTT- Mocupe). Este lugar de estudio comprende 650 hectáreas, de las cuales 50 se encuentran con riego por goteo y esté ubicado a la orilla izquierda del kilómetro 02 de la carretera Mocupe - Zaña, que parte del sitio denominado Cruce de Zaña, ubicado en la margen derecha del kilómetro 745 de la carretera Panamericana Norte y a cinco minutos en auto de la localidad de Mocupe Nuevo.

Coordenadas UTM WGS 84 E: 645,676 / N: 9230,164

\section{Objetivos}

Evaluar la adaptabilidad de la tuna a terrenos eriazos de los desiertos de la costa norte del
Perú, ya que podría ser este cultivo un recurso importante en la economía rural y de las zonas áridas en general, superficies que no cuentan con una precipitación pluvial adecuada para el siembra de especies más exigentes en agua y suelo; toda vez que esta planta es muy eficaz para adaptarse y crecer donde confluyen mayor número de factores limitantes que no son favorables para la mayoría de especies vegetales.

Nuestra experiencia se inició considerando los estándares nacionales y a partir de esta línea de base establecer comparaciones y medir los resultados. El tiempo de vida promedio de los cultivos de tuna a nivel comercial es de 5 a 7 años, alcanzando algunas veces hasta 10 años en terrenos apropiados, con $\mathrm{pH}$ Neutro y con labores culturales permanentes. Con un buen manejo de plagas y labores oportunas la planta de tuna puede llegar a vivir hasta 80 años alcanzando de 80 a $90 \mathrm{TM} / \mathrm{ha} /$ año de masa fresca. Sus características morfológicas y fisiológicas particulares, les confieren una notable capacidad de adaptación a los ambientes más hostiles, donde se multiplican y desarrollan fácilmente, proporcionando una productividad más alta que la de muchas de las actuales plantas cultivadas. Sin duda, ello permite que este cultivo sea de fácil adopción por los productores, brindando tanto una alternativa económica y social de producción, como más oportunidades de ocupación en el medio.

\section{MATERIALES Y MÉTODOS}

La siembra se ha realizado a partir del mes de marzo del 2013 en dos hectáreas de terreno de características agrícolas muy pobres (Tabla 1).La densidad de siembra considerando el terreno y la tecnología aplicada se ha hecho con distanciamiento de tres metros entre hileras y un metro entre plantas, haciendo una población estimada de 3333 plantas por hectárea. 
Para la siembra de cada paleta, desinfectada previamente con sulfato de cobre, se aplicó 15 kilos de compost por planta en pozos pequeños de $0,40 \mathrm{~m}$ de profundidad y $0,40 \mathrm{~m}$ de diámetro, con la finalidad de mejoramiento del suelo, ya que éste tiene cero restos orgánicos, y retener humedad y nutrientes.

Tabla 1. Análisis de suelo: Fertilidad. (*) Pampa de San Nicolás

$\begin{array}{cccccc}\text { CE } & \mathrm{pH} & \mathrm{CaCo3} & \mathrm{MO} & \mathrm{P} & \mathrm{K} 20 \\ \mathrm{DS} / \mathrm{m} & & \% & \% & \mathrm{Ppm} & \mathrm{KG} / \mathrm{HA} \\ 0.17 & 8.4 & 2.28 & 0.28 & 3.10 & 404\end{array}$

$\left(^{*}\right)$ Laboratorio de la Universidad Nacional Agraria La Molina. Julio 1998

Al término de cada cosecha se realizaron podas de formación para que las calles no se cierren y las plantas no crezcan demasiado altas; así como luego de la producción, se cortaron las pencas terminales con el objetivo de detener la producción, logrando que las reservas se acumulen para la próxima campaña.

\section{Riego}

El sistema de riego es por goteo, instalado con mangueras ciegas de $16 \mathrm{~mm}$ de diámetro, en las cuales se colocó un micro tubo de 18 litro/hora por planta, teniendo una frecuencia de riego de dos horas diarias por semana, lo que hace un gasto de agua anual de 1872 litros/planta.

El consumo de agua por hectárea está en función a la densidad, es decir a la distancia entre hileras $(3 \mathrm{~m})$ y entre plantas $(1 \mathrm{~m})$.

La falta de agua no nos permitió hacer una siembra a doble hilera que nos permitiría duplicar el número de plantas.

\section{RESULTADOS}

Se ha obtenido un rendimiento promedio de 42 frutos por planta, (seis unidades kilo) lo que hace un promedio de 23000 kilos por cosecha/ hectárea.
El ciclo de desarrollo de los frutos de tuna, a partir del inicio de floración en la planta hasta su completa maduración y el punto máximo de desarrollo y madurez fisiológica de los frutos, se obtiene a los 80 días después de la antesis, teniendo una calidad de fruto con un peso de 170 gramos promedio y una coloración roja intensa.

Por los altos rendimientos y la calidad nutricional y medicinal de los frutos, así como los resultados obtenidos por nuestras investigaciones experimentales, la tuna en estas localidades podría convertirse en un cultivo potencial para la exportación

\section{DISCUSIÓN}

Dentro delos parámetros de producción y calidad de la fruta que se obtiene en desiertos (como el del Centro de Investigación, Producción y transferencia Tecnológica - Mocupe, de la Universidad Alas Peruanas) y la escasez de agua, se llega a obtener un producto de calidad con un rendimiento que está dentro de los estándares de producción aceptables y comerciables, y que sería una nueva opción para la agricultura costeña del norte del país.

En este contexto, para favorecer la producción nacional de tuna es necesario capacitar a los 
productores en la aplicación de innovación tecnológica, así como en la integración de organizaciones o asociaciones que les permita mejorar los canales de comercialización y, con ello, acceder a otros mercados.

Otra vertiente de acción sería la obtención de productos con un mayor valor agregado, mediante la industrialización. Asimismo, se debe impulsar la venta directa a los grandes centros comerciales y tiendas de autoservicio, lo que requiere contar con la suficiente infraestructura de acopio, empaque, almacenamiento y distribución.

\section{BIBLIOGRAFÍA}

1. Griffth, P. 2004 "The origins of an important cactus crop, Opuntia ficus-indica (Cactaceae) new molecular evidence" . American Journal of Botany 91 (11) 1915 - 1921.

2. Mostacero, J., Mejía, F. Gamarra, O. 2002 Taxonomía de la fanerógamas útiles del Perú. Vol I - II, Concytec, Trujillo, Perú

Figura 1

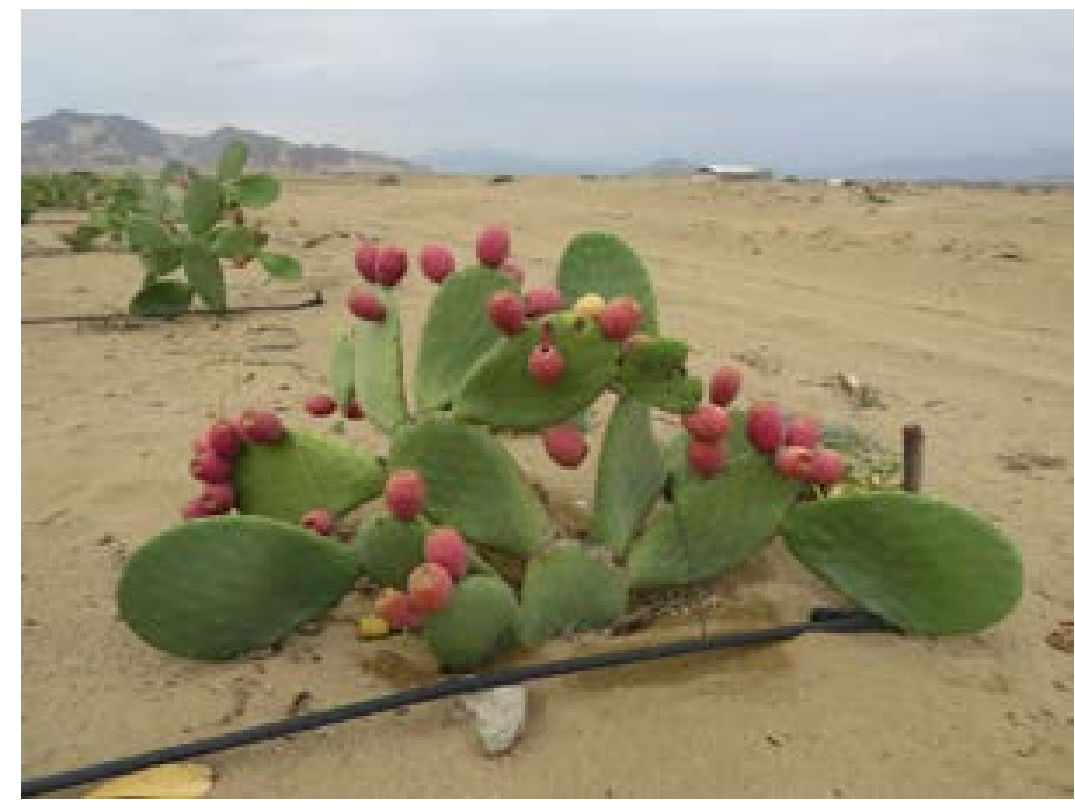

Figura 2

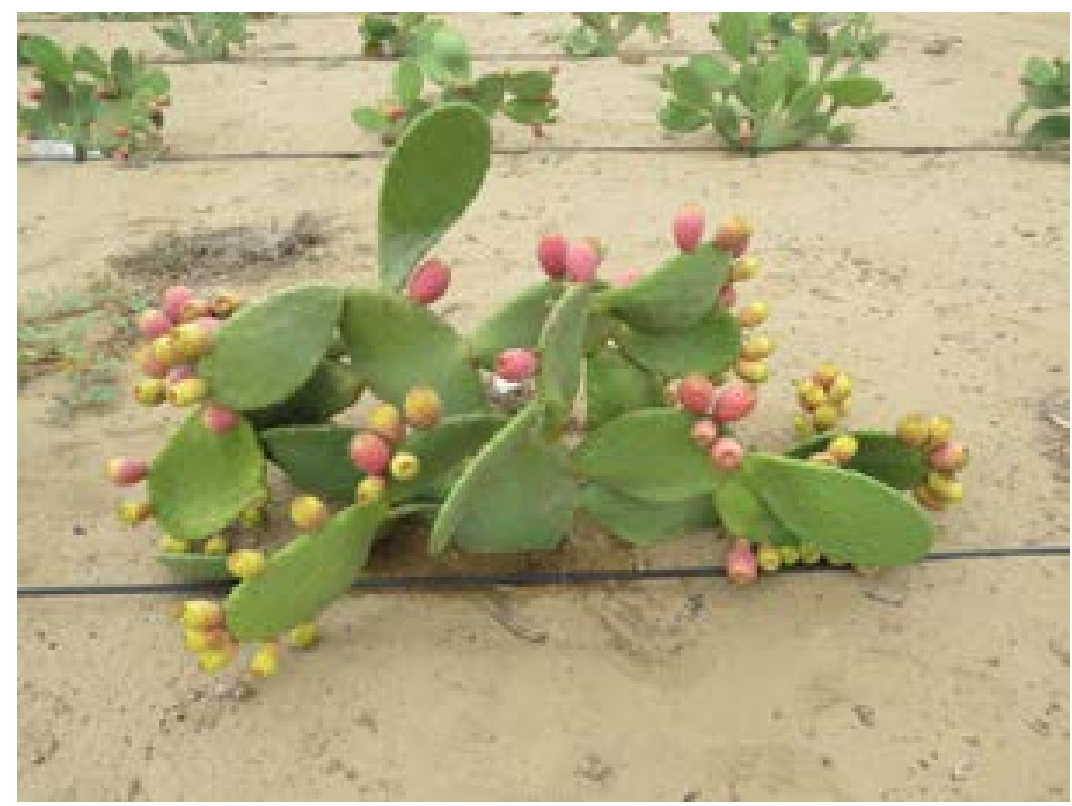

